chain of hypercyclic interactions taking place in an inhomogeneous medium that is represented by a square matrix of 300 times 300 (or 90,000) cells. Each cell, in any one generation, contains either a molecule of a particular species or is empty. The molecular chemistry of the hypercycle works itself out through processes of decay (the cell becomes empty), replication (a molecule in a cell can replicate into an empty neighbouring cell) and catalysis (the probability of such replication is increased if there are catalytic molecules in at least one adjacent cell). Movement or diffusion among neighbouring cells operates in between each generational 'time step', according to rules that are standard for such cellular automaton models ${ }^{5,6}$.

In a manner that is becoming increasingly familiar (since first studied in models of the Belousov-Zhabotinski reaction $)^{5-9}$, Boerlijst and Hogeweg find that their model gives spiral waves that continually move outward from foci that themselves shift over time, giving rise to rich patterns as spiral waves move and collide. Boerlijst and Hogeweg also give an insightful account of the biology of what is going on. Within the spiral, there is a very unequal distribution of darwinian fitness, with the molecules in the middle of the spiral generating the eventual offspring of the entire spiral (in cyclic sequences of molecular species), whereas the molecules at the edge of the spiral disappear. That is, the spatial self-structuring gives rise to a small sub-class of molecules which dominates reproduction.

When a parasitic molecular species of the Maynard Smith variety appears in this spatially structured world, its fate is very different from the selfish success it would enjoy - to the eventual destruction of the entire system - in a homogeneous setting. Instead, Boerlijst and Hogeweg show in detail that such parasitic populations necessarily grow in the direction of the outward movement of the spirals, and thereby are destroyed. Thus parasites appear, and successfully grow, but always toward the outer periphery of the spirals, where all molecules are extinguished. This conclusion is robust, deriving from the spatial structure of the system: Boerlijst and Hogeweg show that even parasites with very large replicative advantages are readily purged soon after they appear. The only exceptions arise if a parasite originates exactly at the centre of a spiral; in this event the parasite can survive as a relatively small 'cyst', encapsulated at the spiral's centre (and if it ever escapes, then it is swept to the spiral's edge and removed).

Ever since the rich and varied patterns generated by John Horton Conway's Game of Life (based on a cellular auto- maton) were brought to the attention of a wide audience by Martin Gardner's column in Scientific American, many scientists have been fascinated by cellular automata as metaphors for living things. As surveyed by Langton ${ }^{10}$ and others (mostly with backgrounds in physics), a growing literature is based on the view that the self-structured patterns seen in cellular automaton models may help us understand prebiotic replicators, and thence early evolutionary processes. These studies of cellular automata are a lot of fun, but I do not believe they are likely to tell us much about the origins of life, any more than do ordinary crystals (self-structured objects on large scales).

Boelijst and Hogeweg's work is something altogether different. Unlike the work of Langton and others who interpret the spontaneous self-structuring in cellular automata as a form of evolution, Boelijst and Hogeweg see this selfstructuring as a "substrate for selection". Their work has specific importance in bringing hypercycles back into play as mechanisms that may facilitate prebiotic evolution; the self-generated and deterministic spatial structure in their models provides an environment within which interspecific group selection can operate in a robust way, ridding the system of parasitic molecules. More generally, the work is important in highlighting the need to examine evolutionary and ecological problems not in homogeneous environments, but in spatially structured environments that themselves can be generated by simple rules. As in most previous studies, Boelijst and Hogeweg consider a situation where the selfgenerated spatial structure consists of spiral waves, but many other patterns, including deterministically generated chaos $^{6}$, can arise. It is not clear to me whether Boelijst and Hogeweg's models can ever generate chaotic substrates, nor what the consequences would be if they could, but my guess is that these kinds of questions are likely to be a growth industry.

Robert M. May is in the Department of Zoology, University of Oxford, South Parks Road, Oxford OX1 3PS, UK.

1. Eigen, M. \& Schuster, P. The Hypercycle: A Principle of Natural Self-Organisation (Springer, New York, 1979) Maynard Smith, J. Nature 280, 445-446 (1979)

3. Boerlijst, M. C. \& Hogeweg, P. Physica D48, 17-28 (1991).

4. Nowak, M. \& Schuster, P. J. theor. Biol. 137, 375-395 (1989).

5. Gerhardt, M., Schuster, H. \& Tyson, J. J. Science 247 1563-1565 (1990)

6. Hassel, M. P., Comins, H. N. \& May, R. M. Nature 353 255-258 (1991)

Winfree, A. T. When Time Breaks Down (Princeton University Press, 1987)

8. Muller, S. C. Plesser, T. \& Hess, B. Physica D24, 71-85 (1987)

9. Keener, J. P. \& Tyson, J. J. Physica D21, 307-322 (1988)

10. Langton, C. (ed.) Artificial Life: Studies in the Science of Complexity (Addison-Wesley, New York, 1988).

\section{Falsified teeth}

OF all parts of the living human body, the teeth are most nearly dead. Their metabolic turnover is almost nil; so are their powers of healing or regeneration. Like dead wood, they submit passively to decay. They tolerate implants, like fillings and crowns, with no reaction at all. Their sole concession to awareness is the ability to feel pain.

More exasperating still, tooth-pain doesn't even warn of impending damage. It is futilely and pointlessly stimulated by the movement of fluid in the tiny tubules that run through the dentine. Heat, cold, drying or the osmotic pressure of sugarbased foods can all set up this flow and cause pain; decay does not. Tooth owners, says Daedalus, have every right to be aggrieved.

Structurally, teeth are inorganic apatite bonded and toughened by a cunning interweaving of organic protein. Toothdecay attacks this protein. In this connection Daedalus recalls the traditional method of hardening biological specimens with formaldehyde solution. This crosslinks their protein chains to give an inert, decay-proof, durable solid, chemically reminiscent of melamine resin. Similarly, he argues, treating a tooth with formaldehyde should bind its protein content into a tough, biologically inert and impermeable material - both stopping decay in its tracks, and sealing the pestilential tubules whose permeability permits the tooth to feel pain.

So Daedalus is inventing a cunning dental filling based on polyformaldehyde. This engineering plastic, better known as acetal resin, is kept from depolymerizing back to formaldehyde only by the most ingenious chemistry. A subtly inferior formulation should degrade very slowly, leaking formaldehyde monomer steadily into the dentine beneath. Formaldehyde is decidedly toxic, but can hardly be worse than mercury, a major component of many dental fillings. In any case, the tiny traces leaking from the filling will react almost instantly with the tooth protein. Soon the tooth will be fully crosslinked into a sealed, biologically inert structure, immune to pain and further decay. The polyformaldehyde filling can then be replaced by a more conventional one - perhaps of melamine resin - compatible with the new tooth material and bonding intimately to it.

Thus dentistry will be transformed. At the first hint of trouble, a tooth will not so much be filled with resistant material, as chemically converted into it. Thereafter, it will be totally inert. The long decline which now charts almost everyone's dental history will be arrested. In effect, we will end up wearing our own natural false teeth.

DAVID JONES 